\title{
Identification of molecular biomarkers and pathways of NSCLC: insights from a systems biomedicine perspective
}

\author{
Rakibul Islam', Liton Ahmed', Bikash Kumar Paul ${ }^{1,2,3}$, Kawsar Ahmed $^{2,3^{*}} \mathbb{D}^{\text {, Touhid Bhuiyan }}{ }^{1}$ and \\ Mohammad Ali Moni ${ }^{4}$
}

\begin{abstract}
Background: Worldwide, more than $80 \%$ of identified lung cancer cases are associated to the non-small cell lung cancer (NSCLC). We used microarray gene expression dataset GSE10245 to identify key biomarkers and associated pathways in NSCLC.

Results: To collect Differentially Expressed Genes (DEGs) from the dataset GSE10245, we applied the R statistical language. Functional analysis was completed using the Database for Annotation Visualization and Integrated Discovery (DAVID) online repository. The DifferentialNet database was used to construct Protein-protein interaction (PPI) network and visualized it with the Cytoscape software. Using the Molecular Complex Detection (MCODE) method, we identify clusters from the constructed PPI network. Finally, survival analysis was performed to acquire the overall survival (OS) values of the key genes. One thousand eighty two DEGs were unveiled after applying statistical criterion. Functional analysis showed that overexpressed DEGs were greatly involved with epidermis development and keratinocyte differentiation; the under-expressed DEGs were principally associated with the positive regulation of nitric oxide biosynthetic process and signal transduction. The Kyoto Encyclopedia of Genes and Genomes (KEGG) pathway investigation explored that the overexpressed DEGs were highly involved with the cell cycle; the under-expressed DEGs were involved with cell adhesion molecules. The PPI network was constructed with 474 nodes and 2233 connections.
\end{abstract}

Conclusions: Using the connectivity method, 12 genes were considered as hub genes. Survival analysis showed worse OS value for SFN, DSP, and PHGDH. Outcomes indicate that Stratifin may play a crucial role in the development of NSCLC.

Keywords: Gene expression, Gene ontology, KEGG pathway analysis, PPI network, Molecular biomarkers

\section{Background}

Past few years, lung cancer was taking the leading role in cancer-related death. According to the study, in 2018, worldwide lung cancer was the most common cancer type by contributing 2.01 million diagnosed cases and approximately 1.8 million deaths [1]. Non-small-cell

\footnotetext{
*Correspondence: kawsar.ict@mbstu.ac.bd; k.ahmed.bd@ieee.org; kawsarit08050@gmail.com

${ }^{2}$ Department of Information and Communication Technology, Mawlana Bhashani Science and Technology University, Santosh, Tangail 1902, Bangladesh

${ }^{3}$ Group of Bio-photomatix, Mawlana Bhashani Science and Technology University (MBSTU), Santosh, Tangail 1902, Bangladesh

Full list of author information is available at the end of the article
}

lung carcinoma (NSCLC) is the most common type of lung cancer; more than $80 \%$ of patients with lung cancer were affected by NSCLC [2].. Adenocarcinoma is the most ordinary type of lung cancer; approximately $40 \%$ of NSCLC is adenocarcinoma. This type of NSCLC arises from small airway epithelial, type II alveolar cells, which secrete mucus and other substances [3]. Smoking is listed as one of the worst risk factors for adenocarcinoma [4]. Squamous cell carcinoma (SCC) is the second most common subtype of all lung cancer cases; it constitutes $25-30 \%$ of all lung cancer. SCC is sharply correlated with smoking [5]. 
In recent years, the development in genomics, molecular biology, as well as DNA sequencing methods has guided the identification of many dynamic factors as molecular signature, which may provide better chances for the early detection of cancer [6]. Microarray terminology is referred to as a high-throughput platform used to analyze gene expression and has been broadly used to obtain gene alteration during tumorigenesis and identify prognostic biomarkers in patients with cancer $[7,8]$. In this investigation, we aimed to identify molecular biomarkers for NSCLC using microarray technology, which may help its early diagnosis and prognosis.

In this study, we collected microarray dataset GSE10245 from the Gene Expression Omnibus (GEO) database and utilized $R$ language to identify the differentially expressed genes (DEGs) between adenocarcinoma and SCC. After identifying the DEGs, functional and pathway analysis was performed by using Database for Annotation, Visualization, and Integrated Discovery (DAVID) functional database. To predict the proteinprotein interaction (PPI) network, we used the DifferentialNet repository. The PPI network was visualized by the Cytoscape tool. The Molecular Complex Detection (MCODE) technique was fruitful to perform module analysis from the constructed PPI network. We calculate connectivity degree value to identify hub genes. After that, the overall survival (OS) analysis was done by using the Kaplan-Meier (KM) plotter. The goal of this investigation is to identify molecular biomarkers, to make potential therapeutic medicine for future NSCLC treatment. Figure 1 shows the flow chart of the present study.

\section{Methods}

\section{Gene expression profile data}

Selected microarray gene expression profile GSE10245 was collected from the NCBI's GEO (http://www.ncbi. nlm.nih.gov/geo/) repository $[9,10]$. The GPL570 platform with Affymetrix Human Genome U133 Plus 2.0 Array was used for the dataset. Fifty-eight NSCLC-associated samples were found in GSE10245.

\section{Differentially expressed gene (DEG) screening}

The selected profile GSE10245 was converted into expression measures using the Linear Models for Microarray and RNA-Seq Data (limma) of the R language [11]. Identified DEGs were collected following the cut-off criteria: $\mid \log$ fold-change (FC) $\mid>1.25$ and $P$ value $<0.05$.

\section{Functional analysis of DEGs}

In the present Bioinformatics analysis, Gene Ontology (GO) analysis is a widely used method to know functional annotation of a gene set [12]. The Kyoto
Encyclopedia of Genes and Genomes (KEGG) database contains genomic information, recognized pathways, gene functions, and gene networks with higher-order functional information of various organisms [13]. The DAVID (http://david.ncifcrf.gov/) is an online tool that can provide wide functional information about genes/ proteins [14]. The present study used the DAVID tool to identify important GO terms and KEGG pathways of identified DEGs.

\section{Protein-protein interaction and module analysis construction}

DifferentialNet repository was used to foretell the potential interaction between gene products in the human lung tissue. The DifferentialNet is a great repository that supplies human organ tissue-specific interactomes information (http://netbio.bgu.ac.il/diffnet/) [15]. Twenty percent of filter interactions were considered as significant. The integration of protein-protein interaction (PPI) networks was constructed by using Cytoscape (Version 3.7.2) [16]. Degree $>5$ was set as the cutoff criteria for the PPI networks. The MCODE algorithm was utilized to identify modules from the PPI network [17]. Additionally, MCODE score $>2$ and amount of node $>10$ were set as cutoff standard to perform the module analysis. After performing the module analysis, we used the DAVID functional database to perform the KEGG pathway analysis of top gene modules. Finally, we identified hub genes based on higher-degree connectivity value in the PPI network.

\section{Survival analysis of hub genes}

The Kaplan-Meier (KM) plotter (http://kmplot.com/ analysis/) online Bioinformatics tool that count the effect of more than 54,000 genes on survival by using around 11,000 samples, including 6234 breast cancer samples, 2190 ovarian cancer samples, 3452 lung cancer samples, and 1440 gastric cancer samples [18]. The overall survival analysis-related information was based on the European Genome-Phenome Archive (EGA), GEO, and The Cancer Genome Atlas (TCGA) database. In the KM plotter, the hazard ratio (HR) and low-rank $P$ value were considered and showed on the plot.

\section{Results}

\section{Differentially expressed genes (DEG) screening}

The GSE10245 gene expression profile was elected in this study. The selected gene expression profile had a total of 58 samples, including 40 ADC samples and 18 SCC samples. Based on criteria $|\log (\mathrm{FC})|>1.25$ and $P$ value $<0.05$, a total of 1082 DEGs were identified from the analyzed dataset, including 419 DEGs were overexpressed and 663 DEGs were under-expressed. 


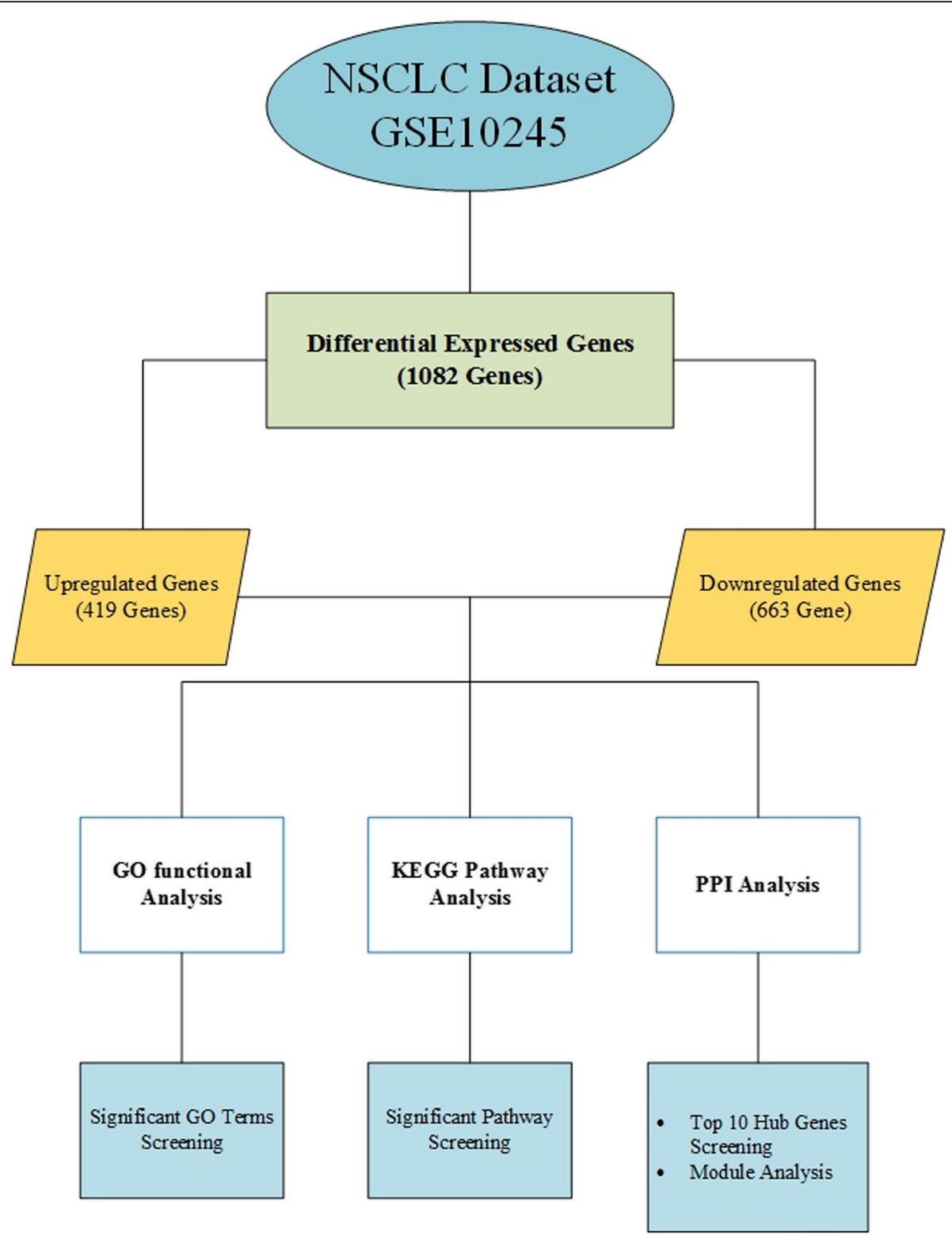

Fig. 1 Flow diagram of the analytical approach used in this investigation

\section{Functional analysis of DEGs}

The GO function terms for DEGs were identified by using the DAVID online database. The overexpressed genes were significantly enhanced in the function of epidermis development, mitotic nuclear division, and keratinocyte differentiation for Biological Process (BP), chromosome, centromeric region and cytoplasm for Cellular Component $(\mathrm{CC})$, and structural molecule activity and microtubule binding for Molecular Function (MF) (Table 1). The under-expressed genes were significantly enhanced in the functions of positive regulation of nitric oxide biosynthetic process and signal transduction for BP, extracellular exosome and extracellular space for $\mathrm{CC}$, and scavenger receptor activity and growth factor activity for MF (Table 2).
In addition, KEGG pathway analyses for the overexpressed and under-expressed DEGs were accomplished using the DAVID database. The overexpressed DEGs were momentously enhanced in chemical carcinogenesis, cell cycle, and Hippo signaling pathway (Table 3), while the under-expressed DEGs were greatly enhanced in complement and coagulation cascades, cell adhesion molecules, and tight junction (Table 4).

\section{PPI and module analysis}

Protein interactions of lung tissue among the identified 1082 DEGs were predicted with the DiffentialNet database. Four hundred seventy four nodes with 2233 connections were attached in the constructed PPI network 
Table 1 The functional analyses of upregulated genes to identify top 15 GO terms

\begin{tabular}{llll}
\hline Category & Term name & Count & P value \\
\hline BP & GO:0008544-epidermis development & 20 & $2.13 \mathrm{E}-14$ \\
BP & GO:0030216-keratinocyte differentiation & 18 & $5.06 \mathrm{E}-13$ \\
BP & GO:0030855-epithelial cell differentiation & 11 & $2.79 \mathrm{E}-06$ \\
BP & GO:0031424-keratinization & 9 & $8.65 \mathrm{E}-06$ \\
BP & GO:0007067-mitotic nuclear division & 19 & $9.58 \mathrm{E}-06$ \\
CC & GO:0001533-cornified envelope & 10 & $4.31 \mathrm{E}-07$ \\
CC & GO:0030057-desmosome & 7 & $8.46 \mathrm{E}-06$ \\
CC & GO:0000775-chromosome, centromeric region & 9 & $2.51 \mathrm{E}-05$ \\
CC & GO:0005737-cytoplasm & 148 & $3.40 \mathrm{E}-05$ \\
CC & GO:0005882-intermediate filament & 11 & $1.45 \mathrm{E}-04$ \\
MF & GO:0005198-structural molecule activity & 24 & $3.60 \mathrm{E}-09$ \\
MF & GO:0008017-microtubule binding & 15 & $1.60 \mathrm{E}-04$ \\
MF & GO:0001758-retinal dehydrogenase activity & 4 & $3.18 \mathrm{E}-04$ \\
MF & GO:0005200-structural constituent of cytoskeleton & 10 & $5.89 \mathrm{E}-04$ \\
MF & GO:0042803-protein homodimerization activity & 30 & 0.001048 \\
\hline BP
\end{tabular}

$B P$ biological process, $C C$ cellular component, $M F$ molecular function

as showed in Fig. 2. In our PPI analysis, we consider the connectivity degree value method to identify hub genes. Connectivity degree values of more than 34 were considered hub genes (Table 5). Outcomes from the PPI network revealed that Estrogen Receptor 1 (ESR1) was the most eminent gene with the highest connectivity degree value (80), followed by AR (degree value $=51$ ), LRRK2 $($ degree value $=45)$, CFTR $($ degree value $=40)$, DSP $($ degree value $=39)$, ZBTB16 (degree value $=39)$, ERBB2 $($ degree value $=38)$, CDK1 $($ degree value $=36)$, EEF1A2
$($ degree value $=36)$, PHGDH (degree value $=35)$, SFN $($ degree value $=35)$, and SOX2 (degree value $=35)$. There were 5 overexpressed and 7 under-expressed genes in identified 12 hub genes.

In this study, the MCODE algorithm was used to identify significant modules by analyzing the constructed PPI network. Thirty one clusters were found using the MCODE algorithm; we identify the top 3 clusters among them (Fig. 3a). The pathway analysis explored that the three modules were principally connected with ErbB

Table 2 The functional analyses of downregulated genes to identify top 15 GO terms

\begin{tabular}{|c|c|c|c|}
\hline Category & Term name & Count & $P$ value \\
\hline CC & GO:0070062_extracellular exosome & 170 & 1.57E-14 \\
\hline CC & GO:0005615—extracellular space & 97 & $3.11 \mathrm{E}-12$ \\
\hline CC & GO:0005886_plasma membrane & 200 & 2.84E-08 \\
\hline CC & GO:0005887_integral component of plasma membrane & 86 & 2.00E-07 \\
\hline CC & GO:0005576—extracellular region & 93 & $5.62 \mathrm{E}-07$ \\
\hline BP & GO:0045429_-positive regulation of nitric oxide biosynthetic process & 9 & $1.02 \mathrm{E}-04$ \\
\hline $\mathrm{BP}$ & GO:0007165—signal transduction & 65 & $1.72 \mathrm{E}-04$ \\
\hline $\mathrm{BP}$ & GO:0050714—positive regulation of protein secretion & 8 & $2.08 \mathrm{E}-04$ \\
\hline $\mathrm{BP}$ & GO:0005975_carbohydrate metabolic process & 17 & 3.93E-04 \\
\hline $\mathrm{BP}$ & GO:0050873_brown fat cell differentiation & 7 & $7.29 \mathrm{E}-04$ \\
\hline MF & GO:0005044—scavenger receptor activity & 8 & 0.001241 \\
\hline MF & GO:0008083—growth factor activity & 14 & 0.004251 \\
\hline MF & GO:0042803_protein homodimerization activity & 40 & 0.005049 \\
\hline MF & GO:0005088—Ras guanyl-nucleotide exchange factor activity & 11 & 0.00664 \\
\hline MF & GO:0003779_actin binding & 19 & 0.008431 \\
\hline
\end{tabular}


Table 3 The pathway analyses of upregulated genes

\begin{tabular}{llll}
\hline Term ID & Term name & Count & P value \\
\hline hsa00980 & Metabolism of xenobiotics by cytochrome P450 & 12 & $1.02 \mathrm{E}-06$ \\
hsa00982 & Drug metabolism_cytochrome P450 & 9 & $1.77 \mathrm{E}-04$ \\
hsa05204 & Chemical carcinogenesis & 9 & $5.47 \mathrm{E}-04$ \\
hsa04110 & Cell cycle & 11 & $6.45 \mathrm{E}-04$ \\
hsa04390 & Hippo signaling pathway & 12 & $8.23 \mathrm{E}-04$ \\
hsa00480 & Glutathione metabolism & 7 & 0.00117 \\
hsa04550 & Signaling pathways regulating pluripotency of stem cells & 9 & 0.01722 \\
hsa04514 & Cell adhesion molecules & 9 & 0.0186 \\
hsa04115 & p53 signaling pathway & 6 & 0.02035 \\
\hline
\end{tabular}

signaling pathway, Prostate cancer, and Viral carcinogenesis (Fig. 3b).

\section{Survival analysis of hub genes}

The KM plotter online experiment tool was used to observe the prognostic values of the identified hub genes. A total of 1926 patient's records were available for the overall survival (OS) analysis. The KM plotter analysis shows that the expression of SFN (HR $=1.59$ [1.4-1.81], low-rank $P=6.5 \mathrm{e}-13$ ) (Fig. 4a) was engaged with worse OS for lung cancer patients, as well as DSP ( $\mathrm{HR}=1.47$ [1.29-1.67], low-rank $P=3.9 \mathrm{e}-09$ ) (Fig. 4b) and PHGD $\mathrm{H}(\mathrm{HR}=1.47$ [1.29-1.66], low-rank $P=2.9 \mathrm{e}-09)$ (Fig. 4c) and identified 12 hub genes mean OS $[\mathrm{HR}=1.45$ [1.23-1.71], low-rank $P=1 \mathrm{e}-05]$ (Fig. 4d).

\section{Discussion}

NSCLC has been a broadly studied topic in cancer research, though there is still a shortage of early detection and diagnosis. Generally, symptoms of NSCLC do not become evident until the cancer is already at an advanced stage; this is one of the principal causes of lacking early detection and diagnosis of NSCLC. Bioinformatics analysis has rapidly increased in the last few years for discovering new therapeutic targets and biomarkers for several cancers [19]. In 2020, Maharjan

Table 4 The pathway analyses of downregulated genes

\begin{tabular}{llll}
\hline Term ID & Term name & Count & $\boldsymbol{P}$ value \\
\hline hsa04610 & Complement and coagulation cascades & 14 & $1.21 \mathrm{E}-06$ \\
hsa04514 & Cell adhesion molecules & 16 & $2.49 \mathrm{E}-04$ \\
hsa05150 & Staphylococcus aureus infection & 8 & 0.003691 \\
hsa04530 & Tight junction & 10 & 0.004956 \\
hsa04974 & Protein digestion and absorption & 10 & 0.005345 \\
hsa05414 & Dilated cardiomyopathy & 9 & 0.01278 \\
hsa04950 & Maturity onset diabetes of the young & 5 & 0.014881 \\
hsa01100 & Metabolic pathways & 59 & 0.025714 \\
hsa00220 & Arginine biosynthesis & 4 & 0.036721 \\
\hline
\end{tabular}

et al., using bioinformatics analysis, identified 16 biomarkers for lung cancer including Cyclin-B2 (CCNB2), Cell Division Cycle 20 (CDC20), F-Box And Leucine Rich Repeat Protein 3 (FBXL3), and Forkhead Box A2 (FOXA2) [20]. Dai et al. identified CDC20, ECT2, MKI67, TPX2, and TYMS as biomarkers using microarray analysis, where Cell Division Cycle 20 (CDC20), Epithelial Cell Transforming 2 (ECT2), Marker of Proliferation Ki-67 (MKI67), TPX2 Microtubule Nucleation Factor (TPX2), and Thymidylate Synthetase (TYMS) showed worse survival outcome [21]. Few studies reveal that Cyclin A2 (CCNA2) and Neuromedin U (NMU) were involved with diagnosis and prognosis of NSCLC $[22,23]$.

In the current study, 1082 DEGs were collected from gene expression dataset GSE10245, including 419 overexpressed DEGs and 663 under-expressed DEGs. The 419 overexpressed genes were significantly enhanced in the function of epidermis development and keratinocyte differentiation for Biological Process (BP), and the 663 downregulated genes were significantly enhanced in the functions of positive regulation of nitric oxide biosynthetic process and signal transduction for BP. The KEGG pathways analysis explored that the overexpressed DEGs were momentously enhanced in Chemical carcinogenesis, Cell cycle, and Hippo signaling pathway, while the under-expressed DEGs were momentously enhanced in Complement and coagulation cascades, Cell adhesion molecules, and Tight junction. The PPI network was constructed with 474 genes and 2233 connections. Twelve genes were deliberated as hub genes including ESR1, AR, LRRK2, CFTR, ZBTB16, DSP, ERBB2, EEF1A2, CDK1, PHGDH, SFN, and SOX2. The top three modules were mainly associated with the ErbB signaling pathway, Prostate cancer, and Viral carcinogenesis. Kaplan-Meier plotter showed that the high expression of 3 out of 12 hub genes was attached with worse OS value, including SFN, DSP, and PHGDH.

Stratifin (SFN) is a member of the 14-3-3 protein family, a highly preserved group of proteins participating by 


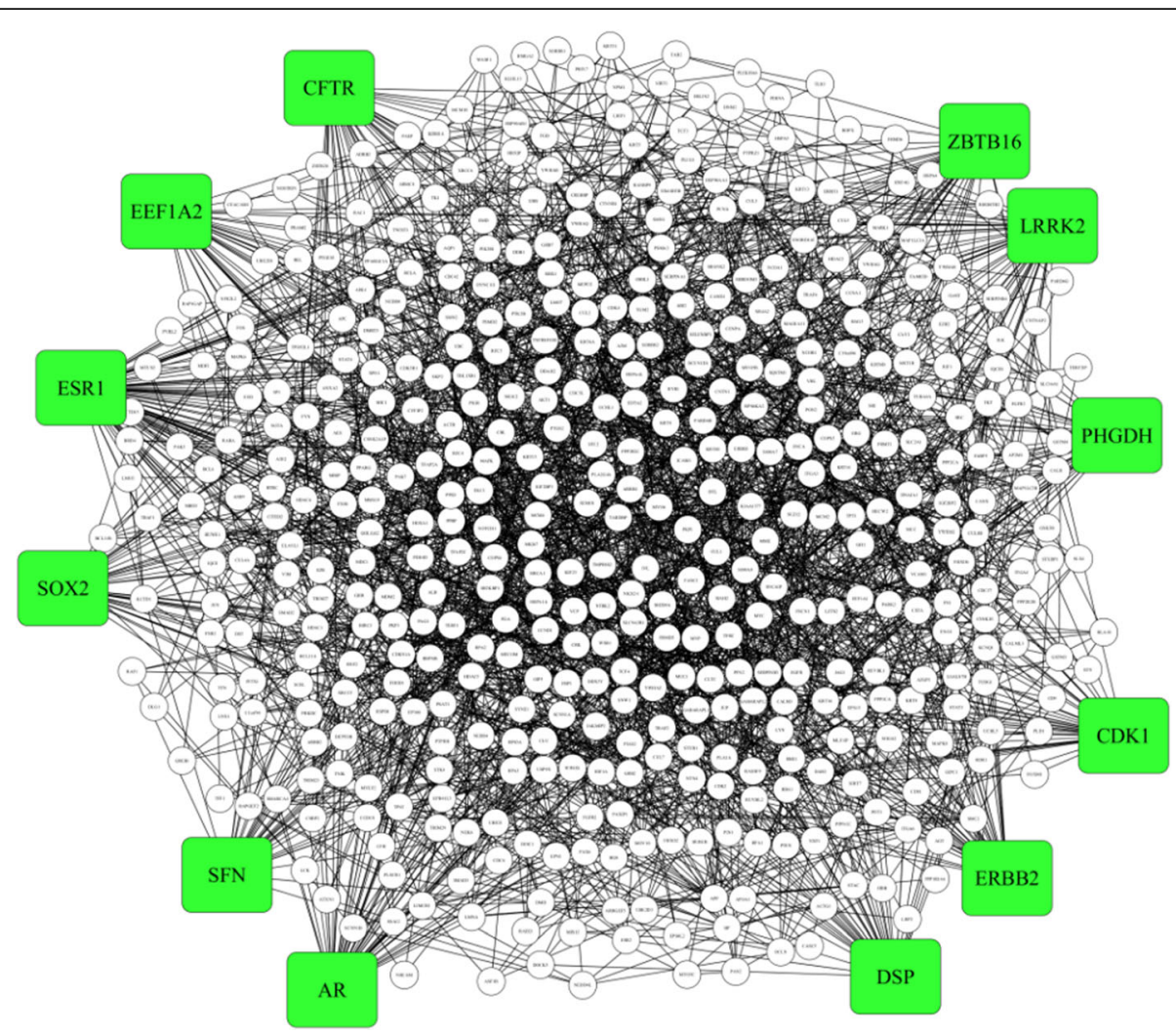

Fig. 2 Protein-protein interaction network with 474 nodes and 2233 connections. Highlighted green color nodes indicate Hub genes of the network

7 isoforms. SFN is engaged with many significant biological functions like cell cycle apoptosis, regulation of signal transduction pathways, and cell proliferation [24, 25].. SFN often plays a role in inhibiting DNA errors during mitosis to respond to DNA damage [26]. SFN had a high expression of malignant progression in earlystage lung adenocarcinoma [24, 27]. Besides, associated with OCIAD2, immunocytochemical staining for SFN could also increase diagnostic sensitivity for lung cancers [28]. SFN gene expression was notably increased and displayed high protein expression in immunohistochemical tarnish of TP53 mutated tumors [29]. In addition, previous study reported that SFN gets involved with multiple kinds of tumor progression including breast, liver, ovarian, and renal tumors [30]. SFN shows also poor OS value in our survival analysis. SFN may play a

Table $\mathbf{5}$ List of hub genes and value of the degree of connectivity

\begin{tabular}{lll}
\hline Gene symbol & Gene name & Degree of connectivity \\
\hline ESR1 & Estrogen receptor 1 & 80 \\
AR & Androgen receptor & 51 \\
LRRK2 & Leucine-rich repeat kinase 2 & 45 \\
CFTR & CF transmembrane conductance regulator & 40 \\
ZBTB16 & Zinc finger and BTB domain-containing 16 & 39 \\
ERBB2 & Erb-B2 receptor tyrosine kinase 2 & 38 \\
EEF1A2 & Eukaryotic translation elongation factor 1 alpha 2 & 36 \\
DSP & Desmoplakin & 39 \\
CDK1 & Cyclin-dependent kinase 1 & 36 \\
PHGDH & Phosphoglycerate dehydrogenase & 35 \\
SFN & Stratifin & 35 \\
SOX2 & SRY-box transcription factor 2 & 35 \\
\hline
\end{tabular}




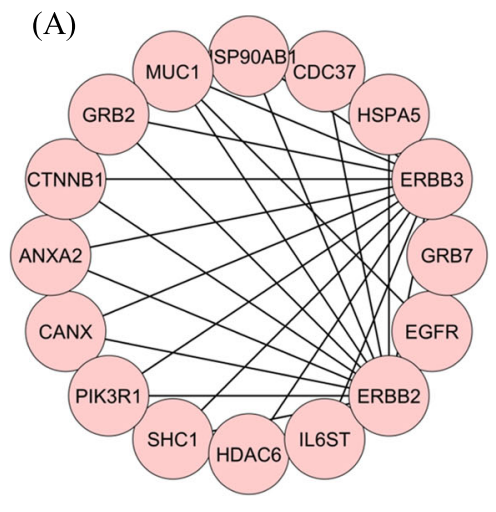

Module 1

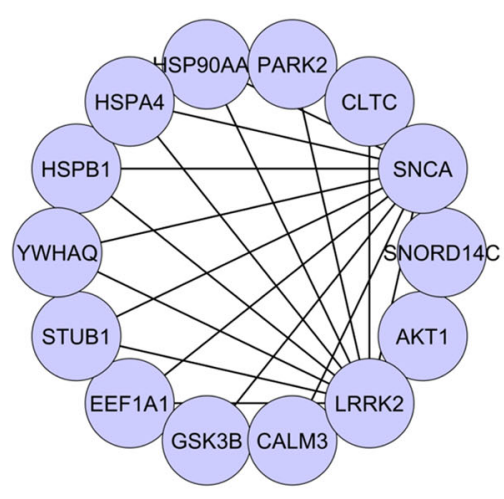

Module 2

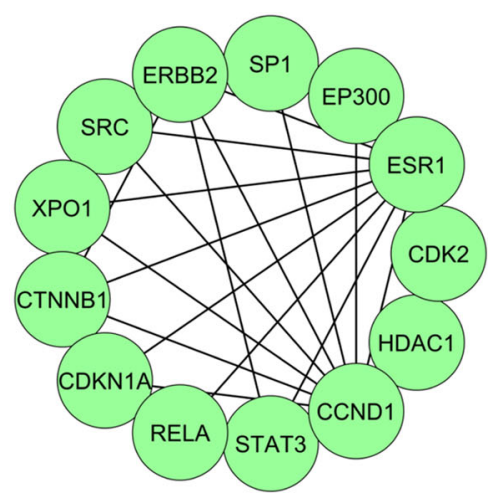

Module 3

(B)

Significant KEGG Pathways of the Top Three Modules

\begin{tabular}{lllll} 
Module & \multicolumn{1}{c}{ Pathway } & P-value & $\begin{array}{c}\text { Adjusted } \\
\text { P-value }\end{array}$ & $\begin{array}{c}\text { Overlapped } \\
\text { Genes }\end{array}$ \\
\hline Module 1 & ErbB signaling pathway & $3.81 \mathrm{E}-11$ & $1.17 \mathrm{E}-08$ & ERBB3;SHC1;ERBB2;GRB2;PIK3R1;EGFR \\
& Prostate cancer & $8.57 \mathrm{E}-11$ & $1.32 \mathrm{E}-08$ & HSP90AB1;ERBB2;CTNNB1;GRB2;PIK3R1;EGFR \\
& Endometrial cancer & $7.33 \mathrm{E}-10$ & $7.52 \mathrm{E}-08$ & ERBB2;CTNNB1;GRB2;PIK3R1;EGFR \\
\multirow{2}{*}{ Module 2 } & Prostate cancer & $3.87 \mathrm{E}-05$ & 0.011926601 & GSK3B;HSP90AA1;AKT1 \\
& Neurotrophin signaling pathway & $7.13 \mathrm{E}-05$ & 0.010974626 & GSK3B;AKT1;CALM3 \\
\multirow{2}{*}{ Module 3 3 Pathways in cancer } & $8.39 \mathrm{E}-05$ & 0.008617744 & GSK3B;HSP90AA1;YWHAQ;AKT1 \\
& & $3.04 \mathrm{E}-16$ & $9.35 \mathrm{E}-14$ & CDKN1A;CCND1;HDAC1;SP1;STAT3;ERBB2;CDK2; \\
& & & & EP300;CTNNB1;ESR1;RELA \\
& Prostate cancer & $8.48 \mathrm{E}-14$ & $1.31 \mathrm{E}-11$ & CDKN1A;CCND1;ERBB2;CDK2;EP300;CTNNB1;RELA \\
& Viral carcinogenesis & $1.12 \mathrm{E}-13$ & $1.14 \mathrm{E}-11$ & CDKN1A;CCND1;HDAC1;SRC;STAT3;CDK2;EP300;RELA
\end{tabular}

Fig. 3 a Top 3 module analysis of PPI network. b Module-associated top significant pathways

vital role in the progression of NSCLC. Estrogen receptor 1 (ESR1) gene plays an active role in the progression of various cancers such as breast, prostate, and endometrial cancer [31-33]. ESR1 gene plays an active role in metastatic breast cancer [34, 35]. Previous report revealed that estrogen receptors (ERs) play significant role in NSCLC progression [36]. ERs might influence several cancer-associated biological functions and pathways in NSCLC, notably, membrane receptor activation and signal transduction, which might ultimately lead the way to changes in cell behaviors. In a recent study, Xiujuan Gao shows that ERs help to develop NSCLC by modulating the membrane receptor signaling network [37]. So ESR1 also may play an active role in the development of NSCL C. Cyclin-dependent kinase 1 (CDK1) covers a vital role in the monitoring of the cell cycle by regulating the centrosome cycle. CDK1 serves as a prognostic biomarker for cancers including colorectal and lung cancers $[38,39]$. Desmoplakin (DSP) is an originating member of the plakin family; DSP is a committed element of desmosomal plaques [40]. Yang et al. showed that DSP acts as a tumor suppressor in lung cancer [41]. The limitations of our study were as follows. First, we use only one dataset. Second, the sample size of the dataset was comparatively small. Third, we could not validate due to the absence of experiments. But we hope our study will make a positive impact to identify biomarkers of NSCLC.

\section{Conclusion}

In summary, we analyzed a microarray dataset GSE10245 of NSCLC and identified 1082 DEGs including 419 upregulated and 663 downregulated DEGs that connected with NSCLC. Functional enrichment analysis explored that overexpressed DEGs were greatly involved with epidermis development and keratinocyte differentiation; the under-expressed DEGs were principally associated with the positive regulation of nitric oxide biosynthetic process and signal transduction. The KEGG pathway analysis showed that the overexpressed DEGs were highly involved with the cell cycle; and the underexpressed DEGs were involved with cell adhesion molecules. From the PPI network analysis, we have found 12 hub genes which has more than or equal 35 connections 


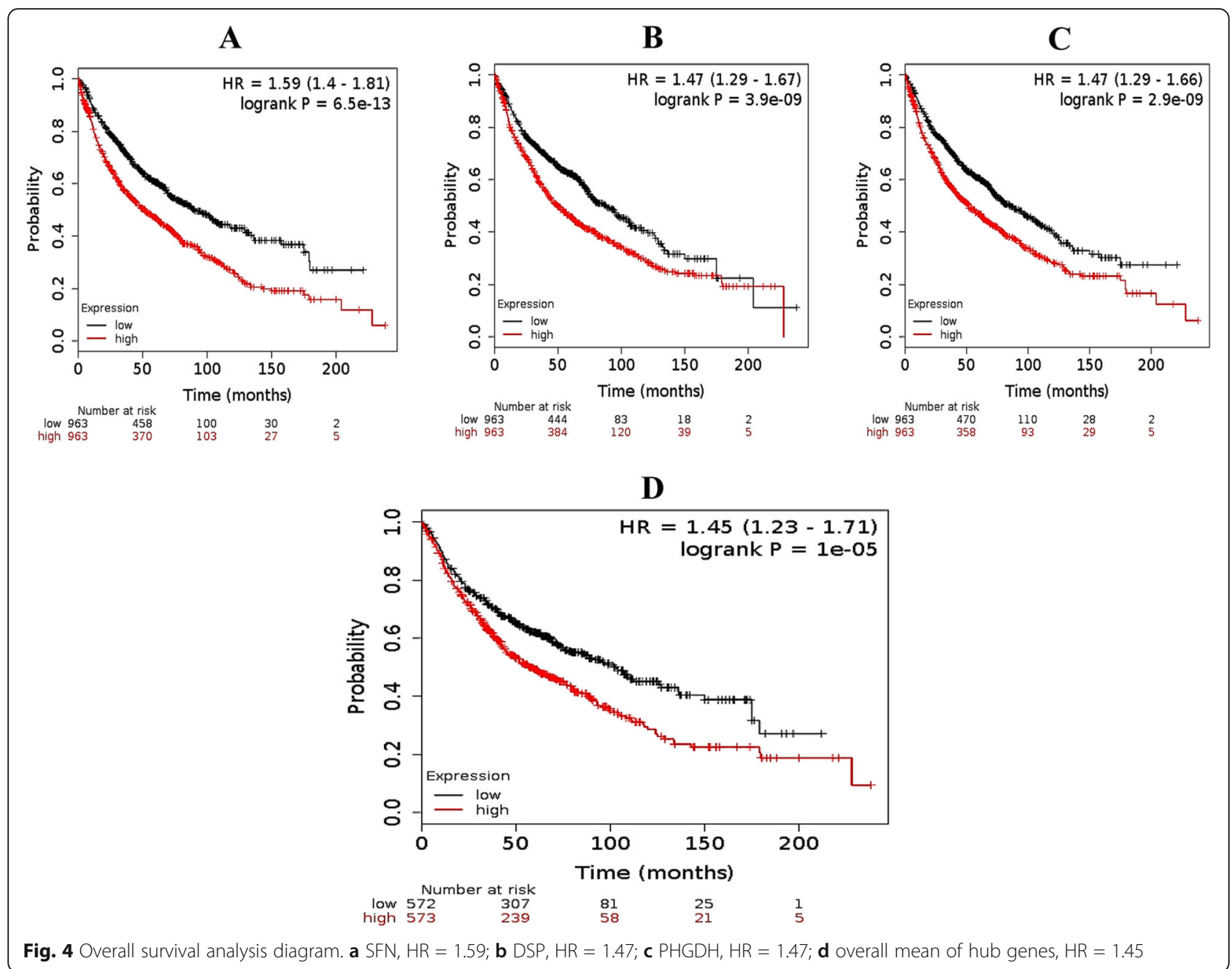

in the network. After implementing the MCODE method, 3 significant clusters were detected, the clusters were mainly connected with ErbB signaling pathway, Prostate cancer, and Viral carcinogenesis. Survival analysis explored that SFN had the worst HR value. Depending on our investigation, we can say that Stratifin (SFN) may play as a biomarker in the progression of NSCLC. Further study needed to confirm our statement.

\section{Acknowledgements}

This manuscript has not been published yet and not even under consideration for publication elsewhere. The authors are grateful who have participated in this research work. We thank the anonymous referees for their useful suggestions.

\section{Abbreviations}

NSCLC : Non-small-cell lung carcinoma; DEGs: Differentially expressed genes; GEO: Gene Expression Omnibus; NCBI: National Center of Biotechnology Information; KEGG: Kyoto Encyclopedia of Genes and Genomes; GO: Gene Ontology; DAVID: Database for Annotation, Visualization, and Integrated Discovery; KM plotter: Kaplan-Meier plotter; MCODE: Molecular Complex Detection; Limma: Linear Models for Microarray and RNA-Seq Data
Authors' contributions

MRI, MLA, and BKP carried out the experimental work and provided the first draft of the manuscript. BKP, KA, TB, and MAM supervised the experimental work and provided manuscript writing assistance. BKP and MAM designed and supervised the work. The authors have read and approved the final manuscript.

\section{Funding}

Not applicable

\section{Availability of data and materials}

The datasets used and/or analyzed during the current study are available from the corresponding author on reasonable request.

Ethics approval and consent to participate

Not applicable

Consent for publication

Not applicable

Competing interests

The authors declare no competing interests.

\section{Author details}

'Department of Software Engineering, Daffodil International University (DIU), Ashulia, Savar, Dhaka 1342, Bangladesh. ${ }^{2}$ Department of Information and Communication Technology, Mawlana Bhashani Science and Technology 
University, Santosh, Tangail 1902, Bangladesh. ${ }^{3}$ Group of Bio-photomatix, Mawlana Bhashani Science and Technology University (MBSTU), Santosh, Tangail 1902, Bangladesh. ${ }^{4}$ WHO Collaborating Centre on eHealth, School of Public Health and Community Medicine, Faculty of Medicine, University of New South Wales, Sydney, Australia.

Received: 7 May 2020 Accepted: 14 February 2021

Published online: 19 March 2021

\section{References}

1. Bray F, Ferlay J, Soerjomataram I, Siegel RL, Torre LA, Jemal A (2018) Global cancer statistics 2018: GLOBOCAN estimates of incidence and mortality worldwide for 36 cancers in 185 countries. CA Cancer J Clin 68(6):394-424

2. Granville CA, Dennis PA (2005) An overview of lung cancer genomics and proteomics. Am J Respir Cell Mol Biol 32(3):169-176

3. Noguchi M, Morikawa A, Kawasaki M, Matsuno Y, Yamada T, Hirohashi S, Kondo H, Shimosato Y (1995) Small adenocarcinoma of the lung. Histologic characteristics and prognosis. Cancer 75(12):2844-2852

4. Subramanian J, Govindan R (2007) Lung cancer in never smokers: a review. J Clin Oncol 25(5):561-570

5. Kenfield SA, Wei EK, Stampfer MJ, Rosner BA, Colditz GA (2008) Comparison of aspects of smoking among the four histological types of lung cancer. Tob Control 17(3):198-204

6. CHEN HY, YU SL, LI KC, YANG PC (2012) Biomarkers and transcriptome profiling of lung cancer. Respirology 17(4):620-626

7. Lu Y, Lemon W, Liu PY, Yi Y, Morrison C, Yang P, Sun Z, Szoke J, Gerald WL, Watson M, Govindan R (2006) A gene expression signature predicts survival of patients with stage I non-small cell lung cancer. PLoS Med 3(12):e467

8. Islam MR, Ahmed ML, Paul BK, Bhuiyan T, Ahmed K, Moni MA (2020) Identification of the core ontologies and signature genes of polycystic ovary syndrome (PCOS): A bioinformatics analysis. Inform Med Unlocked 18: 100304

9. Kuner R, Muley T, Meister M, Ruschhaupt M, Buness A, Xu EC, Schnabel P, Warth A, Poustka A, Sültmann H, Hoffmann H (2009) Global gene expression analysis reveals specific patterns of cell junctions in non-small cell lung cancer subtypes. Lung Cancer 63(1):32-38

10. Barrett T, Wilhite SE, Ledoux P, Evangelista C, Kim IF, Tomashevsky M, Marshall KA, Phillippy KH, Sherman PM, Holko M, Yefanov A (2012) NCBI GEO: archive for functional genomics data sets-update. Nucleic Acids Res 41(D1):D991-D995

11. Smyth GK, Ritchie M, Thorne N, Wettenhall J (2005) LIMMA: linear models for microarray data. In: Bioinformatics and Computational Biology Solutions Using R and Bioconductor. Statistics for Biology and Health

12. Ashburner M, Ball CA, Blake JA, Botstein D, Butler H, Cherry JM, Davis AP, Dolinski K, Dwight SS, Eppig JT, Harris MA (2000) Gene ontology: tool for the unification of biology. Nat Genet 25(1):25-29

13. Kanehisa M, Goto S (2000) KEGG: kyoto encyclopedia of genes and genomes. Nucleic Acids Res 28(1):27-30

14. Sherman BT, Lempicki RA (2009) Systematic and integrative analysis of large gene lists using DAVID bioinformatics resources. Nat Protoc 4(1):44

15. Basha O, Shpringer R, Argov CM, Yeger-Lotem E (2018) The DifferentialNet database of differential protein-protein interactions in human tissues. Nucleic Acids Res 46(D1):D522-D526

16. Shannon P, Markiel A, Ozier O, Baliga NS, Wang JT, Ramage D, Amin N, Schwikowski B, Ideker T (2003) Cytoscape: a software environment for integrated models of biomolecular interaction networks. Genome Res 13(11):2498-2504

17. Bader GD, Hogue CW (2003) An automated method for finding molecular complexes in large protein interaction networks. BMC Bioinformatics 4(1):1-27

18. Győrffy B, Surowiak P, Budczies J, Lánczky A (2013) Online survival analysis software to assess the prognostic value of biomarkers using transcriptomic data in non-small-cell lung cancer. PLoS One 8(12):e82241

19. Jiang P, Liu XS (2015) Big data mining yields novel insights on cancer. Nat Genet 47(2):103-104

20. Maharjan M, Tanvir RB, Chowdhury K, Duan W, Mondal AM (2020) Computational identification of biomarker genes for lung cancer considering treatment and non-treatment studies. BMC Bioinformatics 21(9):1-19

21. Dai B, Ren LQ, Han XY, Liu DJ (2020) Bioinformatics analysis reveals 6 key biomarkers associated with non-small-cell lung cancer. J Int Med Res 48(3): 0300060519887637
22. Xiao Y, Feng M, Ran H, Han X, Li X (2018) Identification of key differentially expressed genes associated with non-small cell lung cancer by bioinformatics analyses. Mol Med Rep 17(5):6379-6386

23. You S, Gao L (2018) Identification of NMU as a potential gene conferring alectinib resistance in non-small cell lung cancer based on bioinformatics analyses. Gene 678:137-142

24. Shiba-Ishii A, Kim Y, Shiozawa T, lyama S, Satomi K, Kano J, Sakashita S, Morishita Y, Noguchi M (2015) Stratifin accelerates progression of lung adenocarcinoma at an early stage. Mol Cancer 14(1):1-6

25. Medina A, Ghaffari A, Kilani RT, Ghahary A (2007) The role of stratifin in fibroblast-keratinocyte interaction. Mol Cell Biochem 305(1):255-264

26. Rizou M, Frangou EA, Marineli F, Prakoura N, Zoidakis J, Gakiopoulou H, Liapis G, Kavvadas P, Chatziantoniou C, Makridakis M, Vlahou A (2018) The family of 14-3-3 proteins and specifically 14-3-30 are up-regulated during the development of renal pathologies. J Cell Mol Med 22(9):4139-4149

27. Shiba-Ishii A, Kano J, Morishita Y, Sato Y, Minami Y, Noguchi M (2011) High expression of stratifin is a universal abnormality during the course of malignant progression of early-stage lung adenocarcinoma. Int J Cancer 129(10):2445-2453

28. Itoguchi N, Nakagawa T, Murata Y, Li D, Shiba-Ishii A, Minami Y, Noguchi M (2015) Immunocytochemical staining for stratifin and OCIAD 2 in bronchial washing specimens increases sensitivity for diagnosis of lung cancer. Cytopathology 26(6):354-361

29. Kudo I, Esumi M, Kusumi Y, Furusaka T, Oshima T (2017) Particular gene upregulation and p53 heterogeneous expression in TP53-mutated maxillary carcinoma. Oncol Lett 14(4):4633-4640

30. Hu Y, Zeng Q, Li C, Xie Y (2019) Expression profile and prognostic value of SFN in human ovarian cancer. Biosci Rep 39(5)

31. Reinert T, Coelho GP, Mandelli J, Zimermann E, Zaffaroni F, Bines J, Barrios CH, Graudenz MS $(2019,2019)$ Association of ESR1 mutations and visceral metastasis in patients with estrogen receptor-positive advanced breast cancer from Brazil. J Oncol

32. Lebeau A, Grob TJ, Holst F, Seyedi-Fazlollahi N, Moch H, Terracciano L, Turzynski A, Choschzick M, Sauter G, Simon R (2008) Oestrogen receptor gene (ESR1) amplification is frequent in endometrial carcinoma and its precursor lesions. J Pathol 216(2):151-157

33. Wang YM, Liu ZW, Guo JB, Wang XF, Zhao XX, Zheng X (2013) ESR1 gene polymorphisms and prostate cancer risk: a HuGE review and meta-analysis. PLoS One 8(6):e66999

34. Lei JT, Gou X, Seker S, Ellis MJ (2019) ESR1 alterations and metastasis in estrogen receptor positive breast cancer. J Cancer Metastasis Treat 5

35. Clatot F, Perdrix A, Beaussire L, Lequesne J, Lévy C, Emile G, Bubenheim M, Lacaille S, Calbrix C, Augusto L, Guillemet C (2020) Risk of early progression according to circulating ESR1 mutation, CA-15.3 and cfDNA increases under first-line anti-aromatase treatment in metastatic breast cancer. Breast Cancer Res 22:1-12

36. Hemnes AR (ed) (2015) Gender, Sex Hormones and Respiratory Disease: A Comprehensive Guide. Humana Press. https://doi.org/10.1007/978-3-319-23998-9

37. Gao X, Cai Y, Wang Z, He W, Cao S, Xu R, Chen H (2019) Estrogen receptors promote NSCLC progression by modulating the membrane receptor signaling network: a systems biology perspective. J Transl Med 17(1):1-15

38. Li J, Wang Y, Wang X, Yang Q (2020) CDK1 and CDC20 overexpression in patients with colorectal cancer are associated with poor prognosis: evidence from integrated bioinformatics analysis. World J Surg Oncol 18(1):1-11

39. Li M, He F, Zhang Z, Xiang Z, Hu D (2020) CDK1 serves as a potential prognostic biomarker and target for lung cancer. J Int Med Res 48(2): 0300060519897508

40. Leung CL, Green KJ, Liem RK (2002) Plakins: a family of versatile cytolinker proteins. Trends Cell Biol 12(1):37-45

41. Yang L, Chen Y, Cui T, Knösel T, Zhang Q, Albring KF, Huber O, Petersen I (2012) Desmoplakin acts as a tumor suppressor by inhibition of the Wnt/Bcatenin signaling pathway in human lung cancer. Carcinogenesis 33(10): 1863-1870

\section{Publisher's Note}

Springer Nature remains neutral with regard to jurisdictional claims in published maps and institutional affiliations. 